\title{
NHF18
}

\section{Well Testing and Fracture Network Modeling}

T. W. Doe* (Golder Associates Inc.)

\section{SUMMARY}

This presentation addresses the relationship between fracture network modeling and well test interpretation. Well test provide information on the geometry and properties of fracture networks. Network models can reproduce behaviors similar to conventional well test models, but with different geometries that can have significant impacts on reservoir behavior. Well test interpretations are non-unique, but nonetheless constrained by geometric elements. Further constraints on the well test interpretation must come from geologic and geophysical information, hence well test analysis should be integrated with other disciplines. 


\section{Introduction}

This presentation provides an overview of how well test information can be incorporated into fracture network models. Discrete fracture network models represent reservoirs as networks of planar conductors using geometric data primarily from seismic data and image logs. Meshing the fracture network and applying finite element solution methods provide a basis for determining the impact of the fracture network on reservoir properties and on production. In order to build these models one must have reservoir properties for the individual fractures and not just for the reservoir is a whole.

\section{Background}

Individual fractures are in effect two-dimensional reservoirs whose linkage as a network determines reservoir performance. As such, normal definitions of permeability are not applicable, as each fracture has its own transmissivity or $k h$. Hydraulic diffusivity provides a basis for determining the storage properties of the fracture network. Diffusivity comes from the monitoring of interference responses, which may be either designed well tests or any response over a distance to rates and pressure changes in the reservoir. Mapping the diffusivity responses is the best means of evaluating connectivity and compartmentalization within a fractured reservoir.

The pressure derivative method, which has revolutionized well test interpretation, is fundamental to evaluating the hydraulic geometry of the fracture network. Fracture networks produce derivatives that may appear very similar to those of conventional porous medium conceptual models. The derivative form by itself does not indicate whether or not a reservoir is fractured dominated.

Examples of simple fracture models in this presentation illustrate how fracture networks can produce a wide range of pressure responses. Specific cases show the influence of fracture connectivity in a two-dimensional region, the effect of the dominant fracture orientation, and the influence of faults or other features that provide vertical connectivity across a stratified reservoir. These effects appear as logarithmic straight lines in the derivative plot. As a practical matter, each geometric region, both flow dimensions or boundaries, requires a half to a full log cycle for its identification, and each transition takes up an additional half to full log cycle. Hence, within the timeframe of practical well tests, it is difficult to see more than two or three geometric elements in the well test. Depending on diffusivity, which tends to be large in fracture networks, the well test may see hundreds of meters or more into a reservoir.

\section{Examples}

Examples of well test interpretations from massive carbonates in basement reservoirs provide illustrations of the interpretation approach. These examples show the conventional interpretations from commercial well test analysis programs can be reproduced equally well by fracture network model. The comparison shows that well test interpretations are highly non-unique, however, they are constrained by the observed geometric elements and their effects on transient flow. Once one identifies these elements, which may include channels, planar features, three-dimensional networks, and boundaries, one should seek geological and geophysical evidence to determine what types of real features are consistent with the well test interpretation. Diffusivity plays important role in determining the scale of the well test observation and the types of features that may be influencing the shape of the pressure derivative.

\section{Conclusions}

Well tests provide valuable insights on fracture networks in reservoirs. Discrete fracture network models relate the network geometry to the well test responses. Although well tests are non-unique, the interpretations are nonetheless constrained by the observed geometric elements and other geologic and geophysical information. Well test interpretation should use a highly integrative approach involving multiple disciplines. 